\title{
COMPARATIVE ANALYSIS OF MICROPROCESSORS IN UPPER LIMB PROSTHETICS
}

\author{
Christopher Lake, C.P.O., F.A.A.O.P. \\ Southwest Clinical Director - Advanced Arm Dynamics of Texas \\ John M. Miguelez, C.P., F.A.A.O.P. \\ Senior Clinical Director - Advanced Arm Dynamics, Inc
}

\section{Correspondence should be sent to:}

Christopher Lake, C.P.O., F.A.A.O.P.

Advanced Arm Dynamics of Texas

Center of Excellence

1451 Empire Central

Suite 700

Dallas, Texas 75247 USA

214-631-8200 (phone)

214-631-8208 (fax)

jclake@airmail.net

The recent emergence of microprocessor based prosthetic control for the individual with upper limb deficiency has significantly expanded the spectrum of treatment options and inclusion criteria for this patient population. Microprocessors can accept a wide variety of input devices and ranges enhancing an individual's prosthetic function allowing control options for individuals who were at one time not candidates for such prosthetic management. Additionally, myoelectric control parameters can be adjusted to optimize function while retaining the flexibility to individualize each prosthesis.

\section{Historical Perspective}

Many significant achievements are apparent as one examines the evolution of electronic upper limb prostheses over the last thirty to forty years. The evolution of electronic upper extremity prostheses can be summarized into three distinct generations. First generation electronics, often referred to as digital systems, used an on and off control scheme to actuate electronic terminal devices, wrist rotators, and elbows. These digital systems exhibited a single speed or single rate type of actuation of prosthetic terminal devices. During the first generation there was limited sophistication of input devices. At that time, input devices consisted of myo-electrodes and various switch technology often mounted in the prosthetic interface or attached to a control harness.

Delineation between first and second generation was made at the introduction of the Utah Arm and later the ProControl I prosthetic controller. Both systems allowed for large-scale threshold manipulation, gain or muscle EMG signal amplification as well as adjustment of muscle contraction rate in an attempt to minimize effort required in first generation co-contraction type switching. These systems lower the microvolt 
requirement (by lowering the muscle thresholds) for terminal device, wrist, or elbow control allowing more individuals with upper limb deficiency to take advantage of myoelectric prosthetic technology. Most importantly, these systems introduced proportional control in a reliable electronics package.

Though more sophisticated than the first generation, second generation electronics exhibited challenges that affected the ability of the prosthetist to provide expeditious prosthetic management and interchangeability. Through the second generation, switch activated, single site or dual site myoelectric control systems required different electronic packages. If, during the rehabilitation of the patient, it was noted that dual site type of control was too difficult and single site would be more appropriate, an entire new electronics package would need to be installed into the prosthesis creating additional expense and fabrication time at the point of rehabilitation where timing and expeditious prosthetic function is so very critical.

The third and most current generation of prosthetic electronics incorporates programmable microprocessors. Third generation electronics are delineated by the acceptance of proportional control as the standard. Microprocessors of the third generation allow a more infinite range of adjustment of myoelectric characteristics for the enhancement as well as ease in prosthetic control.

The significant advantage of microprocessor use in upper limb prosthetics is to provide the ability to modify control options (without purchasing or changing components) and input characteristics during initial fitting quickly and easily within the clinical setting. The use of microprocessors allows more complex filtering of the EMG signal resulting in enhanced terminal device responsiveness. Secondarily, microprocessors provide ease in changing control thresholds and sensitivity of the prosthesis as the users strength and ability evolves. Furthermore, microprocessors utilize algorithms to inherently adjust to various situations unknown to the patient. While many related topics are discussed in the literature, there is a void in the literature when one investigates commercially available microprocessor technology (1-9). A significant portion of this paper is based on discussions with individual manufacturers as well as comparative microprocessor analysis performed by the authors in conjunction with the American Academy of Prosthetics and Orthotics Upper Limb Certificate Lecture Series $(10,11)$.

\section{Overview of Microprocessor Comparisons}

Currently, three programmable microprocessors at the transradial and hybrid type levels were reviewed: Otto Bock - Sensor Hand or DMC plus, Motion Control ProControl II, and Liberating Technologies Incorporated - Programmable VariGrip III.

With multiple processors available, it is difficult to identify the appropriate component for a particular patient. Variables that should be examined include the amount of clearance available for microprocessor integration, weight of the microprocessor and appropriate power supply, compatibility of existing components, as well as, the patient requirement for such technology. This comparison of the 
microprocessors provides valuable feedback for the prosthetist as he or she weighs the advantages and disadvantages of each system to optimize both functional and cosmetic requirements for a patient. Fundamental differences were investigated; in particular the characteristics that make each processor unique in clinical application. Objective and subjective comparisons will be explored to discern the fundamental differences between microprocessors.

A rating system approach, commonly utilized in the technology field, provided comparison parameters. A point system was applied to each to provide a means of comparison.

Objective comparisons included:

Provision of written instructions

Weight of the microprocessor

Power supply versatility

Interface Intrusiveness

Control scheme versatility

Warranty guideline of the particular microprocessor

Subjective comparisons included:

Interface method/programming ease

Fabrication specifics

Support Structure

Hybridization - or the ability to incorporate other manufacturers components

Differences noted between the microprocessors indicate that each system possesses unique characteristics that make it attractive or advantageous in specific types of clinical situations. Specific differences will be reviewed at length during the presentation.

\section{Acknowledgements}

Hans Dietl, Otto Bock

Pat Prigge, C.P., Otto Bock

Harold Sears, PhD, Motion Control

William Hansen, MS

T. Wally Williams, MS, Liberating Technology

\section{References}

1 Heckathorne, CW. "Components for Adult Externally Powered Systems", Atlas of Limb Prosthetics, ed by Bowker and Michael, $2^{\text {nd }}$ Ed, Mosby, Chicago (1992) 151-74.

2 Childress, DS "Control of Limb Prostheses", Atlas of Limb Prosthetics, ed by Bowker and Michael, $2^{\text {nd }}$ Ed, Mosby, Chicago (1992) 175-198.

3 Lamb, D. W. "State of the Art in Upper-limb Prosthetics". J of Hand Therapy 
January-March (1993) 1-8.

$4 \quad$ Prosthetics \& Orthotics Engineering, February 1997:1-3.

$5 \quad$ Kyberd, P.J. and Chappell, P.H. “Object-Slip Detection During Manipulation Using A Derived Force Vector”. Mechatronics (1991) 2:1:1-14.

6 Nightingale, J.M. "Microprocessor control of an artificial arm". J of Microcomputer Applications (1985) 8:167-173.

7 Kyberd, P.J., Holland, O.E., Chappell, P.H., Smith, S. Tregidgo, R., Bagwell, P.J., and Snaith, M. "MARCUS: A two Degree of Freedom Hand Prosthesis with Hierarchical Grip Control” IEEE Transactions on Rehab Engineering. (1995) 3:1:70-76.

8 Datta, D. and Brain, N.D. "Clinical Applications of Myoelectrically Controlled Prostheses"

Critical Rev in Phys and Rehab Medicine (1992) 4(3.4): 215-239.

9 Hortensius, P. and Onyshko, S. “A Microcomputer-Based Prosthetic Limb Controller: Design and Implementation”. Annals of Biomedical Engineering. (1987) 15:51-65.

10 Research and related work in conjunction with fellowship lecture "Microprocessors in Upper Limb Prosthetics", Chris Lake, CPO, FAAOP, for the American Academy of Orthotists and Prosthetics Certificate Course in Upper Limb Prosthetics. Including the following discussions initiated in November 2000 which are currently ongoing:

Discussions with Hans Dietl, PhD. Otto Bock, Austria and Pat Prigge, C.P., Otto Bock, USA

Discussions with Harold Sears, PhD, Motion Control

Discussions with William Hansen, MS, and T. Wally Williams, MS, Liberating Technologies

11 Lake, C., Miguelez, JM. "Overview of Microprocessors in Upper Limb Prosthetics," as presented at the ISPO 2001 World Congress in Scotland. 